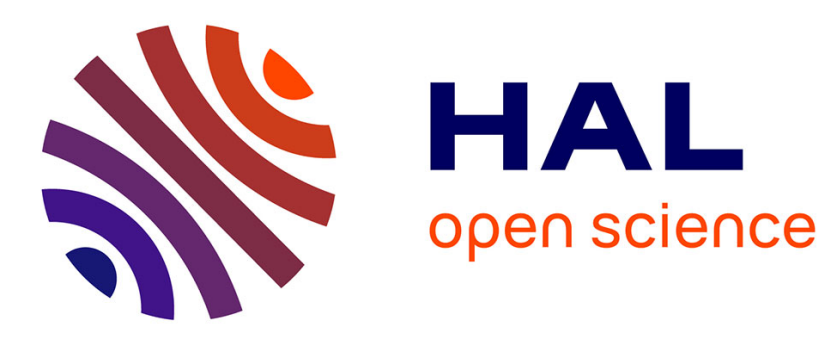

\title{
Domain structure and magnetic anisotropy fluctuations in (Ga,Mn)As : effect of annealing
}

Alexandre Dourlat, Vincent Jeudy, Christophe Testelin, Frédéric Bernardot, Khashayar Khazen, Catherine Gourdon, L. Thevenard, Ludovic Largeau, Olivia Mauguin, Aristide Lemaître

\section{To cite this version:}

Alexandre Dourlat, Vincent Jeudy, Christophe Testelin, Frédéric Bernardot, Khashayar Khazen, et al.. Domain structure and magnetic anisotropy fluctuations in $(\mathrm{Ga}, \mathrm{Mn}) \mathrm{As}$ : effect of annealing. Journal of Applied Physics, 2007, 102 (2), pp.023913. 10.1063/1.2759177 . hal-00166213

\section{HAL Id: hal-00166213 https://hal.science/hal-00166213}

Submitted on 1 Aug 2007

HAL is a multi-disciplinary open access archive for the deposit and dissemination of scientific research documents, whether they are published or not. The documents may come from teaching and research institutions in France or abroad, or from public or private research centers.
L'archive ouverte pluridisciplinaire HAL, est destinée au dépôt et à la diffusion de documents scientifiques de niveau recherche, publiés ou non, émanant des établissements d'enseignement et de recherche français ou étrangers, des laboratoires publics ou privés. 


\title{
Domain structure and magnetic anisotropy fluctuations in (Ga,Mn)As : effect of annealing
}

\author{
A. Dourlat, V. Jeudy, C. Testelin, F. Bernardot, K. Khazen, and C. Gourdon \\ Institut des Nanosciences de Paris, \\ Université Pierre et Marie Curie - Paris 6, \\ Université Denis Diderot - Paris 7, CNRS UMR 7588, \\ 140 rue de Lourmel, 75015 Paris, France
}

L. Thevenard, L. Largeau, O. Mauguin, and A. Lemaître

Laboratoire de Photonique et Nanostructures, CNRS UPR 20

Route de Nozay, 91460 Marcoussis, France

\begin{abstract}
We investigate the effect of post-growth annealing on the magnetic domain structure and magnetization reversal process of $(\mathrm{Ga}, \mathrm{Mn})$ As epilayers grown with tensile strain on a (Ga, In) As buffer. In the case of perpendicular magnetic easy-axis, annealing drastically changes the domain structure observed at magnetization reversal. In as-grown samples, strongly anisotropic domain growth is observed. Dendritic-like domain expansion with guided branching along the $\langle 110\rangle$ directions results in a grid-like pattern. This is tentatively attributed to spatial fluctuations of the uniaxial anisotropy constant, correlated with the cross-hatch pattern. In annealed samples, domain wall motion is much more isotropic, which likely results from a decrease of the relative amplitude of the uniaxial anisotropy fluctuations with increasing carrier density. However domain wall motion is impeded by linear or slightly curved defects, hundreds of micrometers long, and point-like pinning centers. The density of nucleation centers for magnetization reversal strongly decreases upon annealing.
\end{abstract}

PACS numbers: $75.50 . \mathrm{Pp}, 75,60 .-\mathrm{d}, 75.30 . \mathrm{Gw}, 75.60 . \mathrm{Nt}$

\section{INTRODUCTION}

The diluted magnetic semiconductor (Ga,Mn) As has received a lot of attention these past few years as a model system for ferromagnetic semiconductor. ${ }^{1-4}$ Much effort has been devoted to the improvement of the magnetic and electrical properties of epitaxial (Ga,Mn)As layers. ${ }^{5-14}$ Depending on the carrier density and on the strain imposed on $(\mathrm{Ga}, \mathrm{Mn})$ As by the buffer layer, $(\mathrm{Ga}, \mathrm{Mn})$ As epilayers can be prepared with in-plane or perpendicularto-plane magnetic easy-axis. ${ }^{1}$ (Ga,Mn)As samples with in-plane easy-axis, which are promising as spin polariz$\mathrm{ers}^{15}$ and magnetic layers for spin transfer experiments in semiconductor magnetic tunnel junctions ${ }^{16,17}$, are by far the most widely studied. Ferromagnetic layers with perpendicular easy-axis are also of great interest for studying current-induced magnetization reversal, since domain wall switching can be monitored using the polar magnetooptical Kerr effect (MOKE). ${ }^{18}$

The choice for the growth conditions of (Ga,Mn)As layers is limited by the constraint of incorporating a large amount of $\mathrm{Mn}$ atoms while keeping a two-dimensional growth. Post-growth annealing has been found to greatly improve the magnetic and transport properties that are not optimized under the imposed growth conditions. In the recent years, the effect of annealing has been thoroughly investigated in the case of (Ga,Mn)As epilayers with in-plane magnetization. ${ }^{5-12}$ Annealing at a temperature close to the growth temperature was shown to increase the Curie temperature, even up to $173 \mathrm{~K} .^{19}$ This increase was shown to be strongly correlated with a decrease of the resistivity, ${ }^{6-9}$ which results for a large amount from an increase of the carrier density. $5,6,9,10$ Electrical compensation of the $\mathrm{Mn}$ acceptors by donor centers is partially suppressed by annealing. The most important effect was shown to be the out-diffusion of Mn interstitial donor centers. ${ }^{5,12}$ So far, the effect of annealing on the magnetic properties has been mainly discussed in terms of an increase of the Curie temperature. It was also shown that the increase of carrier concentration upon annealing can switch the direction of the magnetic easy-axis from out-of-plane to in-plane for compressive $\operatorname{strain}^{20,21}$ and vice versa for tensile strain ${ }^{14}$. However little is known about the effect of annealing on the magnetic domain structure revealed by imaging.

Imaging of magnetic domain pattems provides information on magnetization reversal processes. Domain nucleation and growth, domain size and spatial organization, domain wall propagation velocity and domain growth anisotropy can be studied. Using optical microscopy based on MOKE with a magneto-optic sensor, large-size domains have been found in (Ga,Mn) As layers with in-plane easy-axis. ${ }^{22}$ From magnetic images obtained using scanning Hall probe (SHP), it was reported that the domain size increases upon annealing for this kind of $(\mathrm{Ga}, \mathrm{Mn})$ As layers. ${ }^{13}$ Small magnetic domains in the micrometer range were observed in as-grown perpendicular-axis (Ga,Mn)As with the use of scanning superconducting quantum interference device (SQUID) and SHP. ${ }^{23,24}$ However, the small size of the scanned area accessible with scanning SQUID and SHP makes it difficult to get information about long-range domain or- 
ganization. Using magneto-optical imaging, which gives access to a much larger field of view $\left(\approx 1 \mathrm{~mm}^{2}\right)$ large-size domains were recently observed in one perpendicular-axis sample after post-growth annealing. ${ }^{14}$ However the effect of annealing on domain size and structure has not been studied.

In this paper, we investigate the effect of post-growth annealing on the magnetic domain structure and magnetization reversal processes for tensile strained $(\mathrm{Ga}, \mathrm{Mn}) \mathrm{As}$ layers, using magneto-optical imaging. In particular we show clear evidence of the influence of the (Ga,In)As buffer on the domain structure for as-grown samples. Section II reports on sample preparation and characterization. Hysteresis cycles obtained from the MOKE images are briefly discussed in Sec. III. Section IV is devoted to the study of the effect of annealing on magnetic domains. Domain growth anisotropy is discussed in Sec. V. Section VI gives a summary and a conclusion.

\section{SAMPLE GROWTH AND CHARACTERIZATION}

\section{A. Samples}

Two samples with different layer thicknesses and buffer compositions were prepared, in order to compare their magnetic domain structure. Their characteristics are displayed in Table I. They were grown by molecular beam epitaxy. Samples 25M05 and 93L06 consist of a $50 \mathrm{~nm}$ and $200 \mathrm{~nm}$ thick $\mathrm{Ga}_{1-x} \mathrm{Mn}_{x}$ As layer, respectively. In a first approximation and without taking into account the cxact location of atoms (on interstitial or substitutional sites $^{25}$ ), the Mn concentration was estimated by high resolution X-ray diffraction. The (Ga,Mn)As layers were grown on a $(\mathrm{Ga}, \mathrm{In}) \mathrm{As}$ relaxed buffer layer deposited on a semi-insulating (001) GaAs substrate. In order to minimize the number of threading dislocations emerging in the magnetic layer, the (Ga,In) As buffer consisted first of a graded layer (thickness $\ell_{1}$ ) with a monotonous increase of the In content from zero to $y$. Then a $\mathrm{Ga}_{1-y} \mathrm{In}_{y} \mathrm{As}$ layer of thickness $\ell_{2}$ was grown. The substrate temperature was set to $400^{\circ} \mathrm{C}$ during the growth of the buffer. The (Ga,Mn)As layers were deposited at $250{ }^{\circ} \mathrm{C}$. The as-grown samples are denoted hereafter as 93L06-ag and $25 \mathrm{M} 05-\mathrm{ag}$. In order to improve the magnetic properties, parts of the samples were annealed. The annealed samples are denoted in the following as 93L06-a and 25M05-a. In the course of sample cutting and processing the absolute orientation of the samples was lost: the $[110]$ and [1]̄0] axes are not distinguished.

The Curie temperature $T_{c}$ was deduced from the temperature dependence of the magnetization obtained using SQUID magnetometry. The magnetic field was along [001]. $T_{c}$ for as-grown and annealed samples is shown in Table I. The low temperature saturation magnetization $M_{s}$ relative to the Mn-carriers spin system is also reported in Table I. It increases upon annealing for sam- ples 25M05 and 93L06.

The out-of-plane and in-plane anisotropies were checked using ferromagnetic resonance (FMR) for samples 25M05-ag and 25M05-a. The FMR spectra were measured with an X-band spectrometer in the first derivative absorption mode, with a modulation frequency of $100 \mathrm{kHz}$. The results are given in the course of the paper.

For magneto-transport measurements, the layers were processed into Hall bars by UV-lithography and wet chemical etching. $\mathrm{Ti} / \mathrm{Au}$ contacts were used. In the absence of magnetic field, these Hall bars were used to obtain four-point resistivity measurements. The results are shown in Fig. 1. Samples 25M05-ag and 93L06-ag both have a metallic character with resistivities in the same range, which suggests that the carrier densities are of the same order of magnitude. After annealing, their resistivity decreases. The peak in the resistivity curve approximately corresponds to the Curie temperature $T_{c}{ }^{6,7,10}$ The critical temperature obtained from resistivity measurements, $56 \mathrm{~K}$ and $120-130 \mathrm{~K}$ for the asgrown and annealed parts of sample 25M05, respectively, are in good agreement with SQUID and MOKE results. A good agreement is also found for sample 93L06. We attribute the decrease of the resistivity and the increase of the critical temperature upon annealing mainly to an increase of hole density, in agreement with Ref. [10].
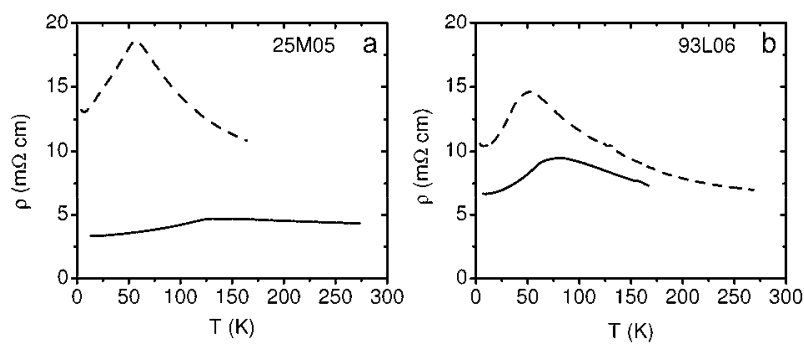

FIG. 1: Thermal dependence of the resistivity for samples 25M05 (a) and 93L06 (b): as-grown (dashed line) and annealed (full line).

\section{B. Surface topography}

For both samples the surface is cross-hatched, showing undulations with grid lines aligned along the $<110>$ crystallographic axes, as commonly encountered in GaInAs/GaAs heterostructures. These undulations, observable by atomic force microscopy (AFM), arise from steps due to the motion of threading dislocations in the $\{111\}$ slip planes. This motion induces the extension of misfit dislocations at the buffer/substrate interface. The gliding of dislocations in the same or in vicinal $\{111\}$ planes leads to the macro-steps. The detailed quantification of steps and the subsequent building mechanism of surface undulations are still a matter of controversy. ${ }^{26-28}$ 
TABLE I: Samples parameters

\begin{tabular}{c|ccccc|c|ccc}
\hline \hline & \multicolumn{2}{|c}{$(\mathrm{Ga}, \mathrm{Mn}) \mathrm{As}$} & \multicolumn{3}{c}{$(\mathrm{Ga}, \mathrm{In}) \mathrm{As}$} & \multicolumn{2}{c}{$T_{c}$} & \multicolumn{2}{c}{$M_{s}\left(\mathrm{kAm} \mathrm{m}^{-1}\right)$} \\
sample & $\mathrm{Mn}$ & $\mathrm{d}(\mathrm{nm})$ & $\ell_{1}(\mu \mathrm{m})$ & $\ell_{2}(\mu \mathrm{m})$ & $\mathrm{In}$ & & as-grown & annealed & as-grown \\
annealed \\
\hline $25 \mathrm{M} 05$ & $7 \%$ & 50 & 0.5 & $2-3$ & $9.8 \%$ & $1 \mathrm{~h} 250^{\circ} \mathrm{C}$ in $\mathrm{N}_{2}$ & 56 & 125 & 28.3 \\
\hline $93 \mathrm{~L} 06$ & $5 \%$ & 200 & $\mathbf{0}$ & $\mathbf{1}$ & $16 \%$ & $50 \mathrm{~h} 190^{\circ} \mathrm{C}$ in air & 55 & 90 & 18.3 \\
\hline \hline
\end{tabular}

In order to characterize the importance of the surface cross-hatch pattern and its possible interplay with the magnetic domain pattern, the surface topography of the samples was investigated by AFM using a Veeco Nanoscope 3100 . The surface undulations of sample 25M05, shown in Fig. 2, have a typical height and spacing of $1-2 \mathrm{~nm}$ and $1-2 \mu \mathrm{m}$, respectively. As seen in Fig. 2, as-grown and annealed pieces of sample 25M05 show the same surface topography, thereby indicating that the post-growth treatment leaves the cross-hatch pattern unchanged. In sample 93L06 surface undulations have a larger typical peak-to-valley amplitude, in the range 5-12 $\mathrm{nm}$. This most probably originates from the higher indium concentration of the buffer layer, leading to a higher density of dislocations. For this sample, the distributions of undulation amplitudes differ along the $\lceil 110\rceil$ and $\lceil 1 \overline{1} 0\rceil$ directions. Along one of these directions, most undulations have a large amplitude with a typical spacing in the range $2-4 \mu \mathrm{m}$. Along the other direction, the distribution of undulation amplitudes is broader, and a typical spacing can hardly be defined. This asymmetry between the $[110]$ and $[1 \overline{10} 0]$ directions was already reported, e.g. in Ref. [28]. It was related to the formation of the $\alpha$ and $\beta$ types of dislocations with different dynamic properties.
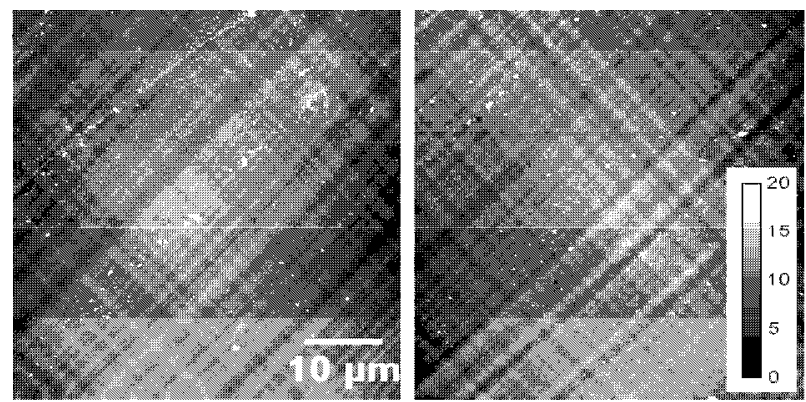

FIG. 2: Surface topography of as-grown (left) and annealed (right) sample 25M05, obtained by atomic force microscopy. The inset in the right image indicates the height scale in $\mathrm{nm}$.

\section{Magneto-optical imaging}

Magneto-optical images were obtained by Kerr microscopy using the intrinsic Kerr effect of the (Ga,Mn) As layer. To the first approximation the MOKE signal is proportional to the perpendicular component of the $\mathrm{Mn}$ magnetization. The cryostat was a variable-temperature, helium-flow Oxford Microstat Hires II pillared cryostat.
The cryostat pillar, onto which the sample was glued, was inserted in the bore of a conventional magnetic coil. The applied field was perpendicular to the sample plane. The temperature could be varied from $3 \mathrm{~K}$ to room temperature. For experiments below $3 \mathrm{~K}$, an immersion-type cryostat was used with the microscope objective placed in the vacuum part of the cryostat. ${ }^{29}$ The optical set-up was similar to a reflection polarizing microscope. The sample was illuminated with light from a halogen lamp filtered at $630(600) \mathrm{nm}$ for sample 25M05 (93L06). The detection system was a CCD camera (Andor DH534-18H-03). The spatial resolution was measured to be $1.5 \mu \mathrm{m}$. Two procedures were used for data acquisition: field cycles or field pulses. In the former case, the field was swept with small increments. After each field step, a delay time was set before image acquisition, in order to allow for field stabilization and fast time evolution of the domain pattern. The acquisition time ( 1 to $3 \mathrm{~s}$ ) was chosen in order to maximize the contrast while avoiding as much as possible time evolution of the domain pattern during acquisition. Faraday rotation from the optics introduced a field-dependent background that had to be removed. In the latter case, starting from the saturated state the field was decreased to zero, then a serie of field pulses of opposite sign and increasing amplitude was applied. The command pulse width was set to $0.4 \mathrm{~s}$, which includes the field-dependent rise time at a speed of $0.6 \mathrm{mT} \mathrm{ms}^{-1}$. At the end of each pulse the field value was back to zero. Owing to domain wall pinning, the magnetic pattern was frozen, which allowed for long acquisition time. Furthermore, by this means, there was no Faraday rotation signal from the optics. This method gave the same result as field sweeping in the case of square hysteresis cycles, but it could not be used when the hysteresis cycle consisted of a double loop. In order to increase the magnetic contrast and to correct for the detector response, raw images were divided by a reference image taken for saturated magnetization. Hysteresis cycles were obtained by plotting the signal averaged over the entire image for each field value. Typical values of the Kerr rotation angle of the samples at saturation of the magnetization were in the range $0.3-1$ degree.

\section{MOKE HYSTERESIS CYCLES}

MOKE hysteresis cycles were obtained for sample 96L06 and sample 25M05. The two samples exhibit different behaviors when the temperature is varied. Sample 93L06-ag has a square hysteresis cycle at all temperatures 
from $1.8 \mathrm{~K}$ to $T_{c}$. This is typical of a sample with perpendicular easy-axis. After annealing the hysteresis cycle remains square. The hysteresis cycles obtained from the MOKE signal for samples 93L06-ag and 93L06-a are displayed in Fig. 3 for temperatures $3 \mathrm{~K}$ and $3.3 \mathrm{~K}$, respectively. Cycles at higher temperatures are very similar, apart from the decrease of the signal amplitude and of the coercive field value with increasing temperature. Post-growth annealing results in small changes of the hysteresis loop: (i) the coercive field slightly increases, at least far from $T_{c}$, and (ii) the magnetization saturation occurs at a larger field. This will be discussed below, in connection with domain structure.

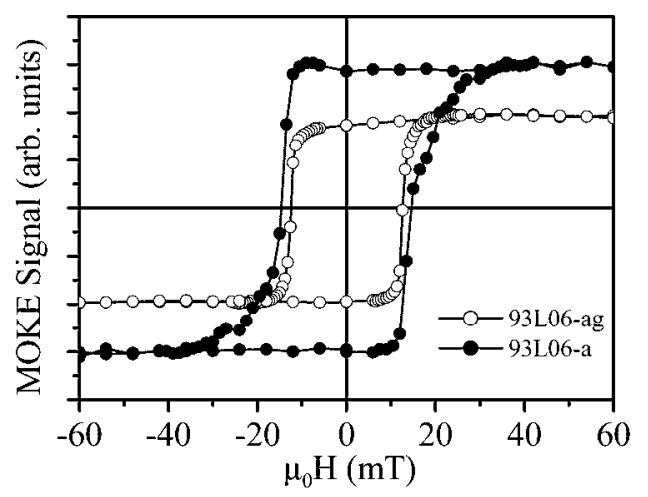

FIG. 3: Hysteresis cycles of the as-grown and annealed samples 93L06-ag and 93L06-a at $3 \mathrm{~K}$ and $3.3 \mathrm{~K}$, respectively, obtained from the MOKE signal.

Contrary to sample 93L06, sample 25M05 shows a striking modification of the hysteresis cycles upon annealing (Fig. 4). Below $\approx 20 \mathrm{~K}$ sample 25M05-ag shows a double loop hysteresis cycle (Fig. 4(a)), which indicates an in-plane easy axis. This is supported by the results of magneto-optical imaging (see Sec. IV). Above $\approx 20$ $\mathrm{K}$ the hysteresis cycle of sample 25M05-ag evolves towards a single square loop, which is the signature of a transition to a perpendicular easy-axis. The annealed sample $25 \mathrm{M} 05$-a shows a square hysteresis loop from $3 \mathrm{~K}$ to the Curie temperature (Fig. 4(b)), consistent with a perpendicular easy-axis. Therefore, below $20 \mathrm{~K}$, annealing induces a reorientation of the magnetic easy-axis from in-plane to perpendicular orientation, which we attribute to the increase of the hole concentration.

Both the carrier-concentration-driven and temperature-driven reorientations of the magnetic easy-axis are in qualitative agreement with the predictions of the mean-field Zener model of carrier-induced ferromagnetism. ${ }^{1}$ Similar temperature-driven reorientation of the magnetic easy-axis from intermediate to perpendicular position was also reported in InMnAs layers grown with tensile strain. ${ }^{30}$ The opposite behavior, i.e. switching from out-of-plane to in-plane magnetic anisotropy upon an increase of carrier concentration or temperature, was reported for $(\mathrm{Ga}, \mathrm{Mn})$ As layers grown under compressive strain. ${ }^{20,21}$
Sample 25M05-a exhibits weaker uniaxial anisotropy than sample 93L06. This likely originates from the lower indium content of the buffer layer and therefore smaller biaxial strain, in agreement with theoretical expectations. $^{1}$

The coercive field $H_{c}$ of sample 25M05-a $\left(\mu_{0} H_{c}=4.4 \mathrm{mT}\right.$ at $\left.3.3 \mathrm{~K}\right)$ is significantly smaller than that of sample 93L 06 -a $\left(\mu_{0} H_{c}=15 \mathrm{mT}\right.$ at $\left.3 \mathrm{~K}\right)$. The precise value of the coercive field depends on the sweeping rate of the applied magnetic field, ${ }^{31}$, but the MOKE hysteresis cycles were taken using similar sweeping rates. This difference in $H_{c}$ is the signature of the existence of different kinds of nucleation/pinning centers in the two samples, as it will be discussed in detail below.
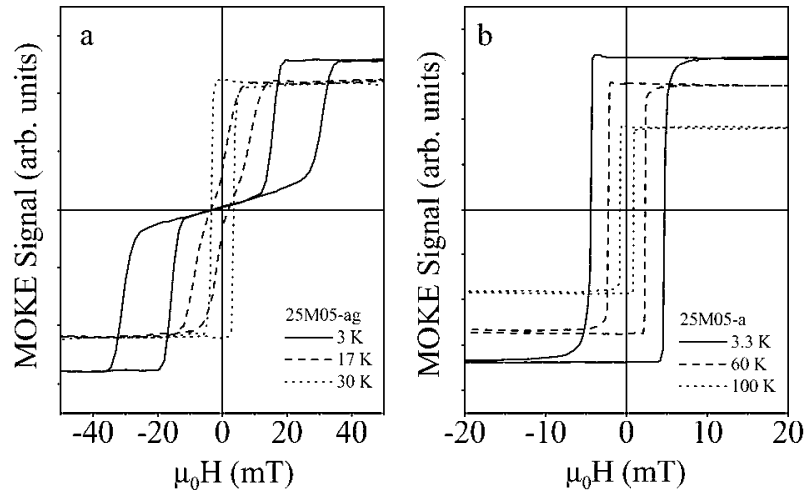

FIG. 4: Hysteresis cycles obtained from MOKE images: (a) as-grown sample 25M05-ag at temperatures $3 \mathrm{~K}, 17 \mathrm{~K}$ and $30 \mathrm{~K}$, (b) annealed sample $25 \mathrm{M} 05$-a at temperatures $3.3 \mathrm{~K}$, $60 \mathrm{~K}$ and $100 \mathrm{~K}$.

\section{DOMAIN STRUCTURE}

\section{A. As-grown samples}

In this section we report on the domain structure of the as-grown samples.

Magneto-optical images are shown in Fig. 5 for sample 93L06-ag, and in Fig. 6 for sample 25M05-ag. They are obtained at temperatures where both samples have perpendicular easy-axis. The images are taken at field values close to the coercive field, as indicated by the arrows on the cycles. For sample 93L06-ag, domains with reverse magnetization first appear as faint stripes, presumably because their width is smaller than the spatial resolution (Fig. 5 (a)). These stripes are aligned along the $<110\rangle$ crystallographic directions. Each isolated stripe results from a single nucleation followed by linear expansion (Fig. 5 (a)). With further sweeping of the field, domain expansion proceeds by branching from one or both sides of the lines. In the early stage this appears as an increase of the line contrast (Fig. 5 (b)). The growth of side branches is very anisotropic, with expansion along the 

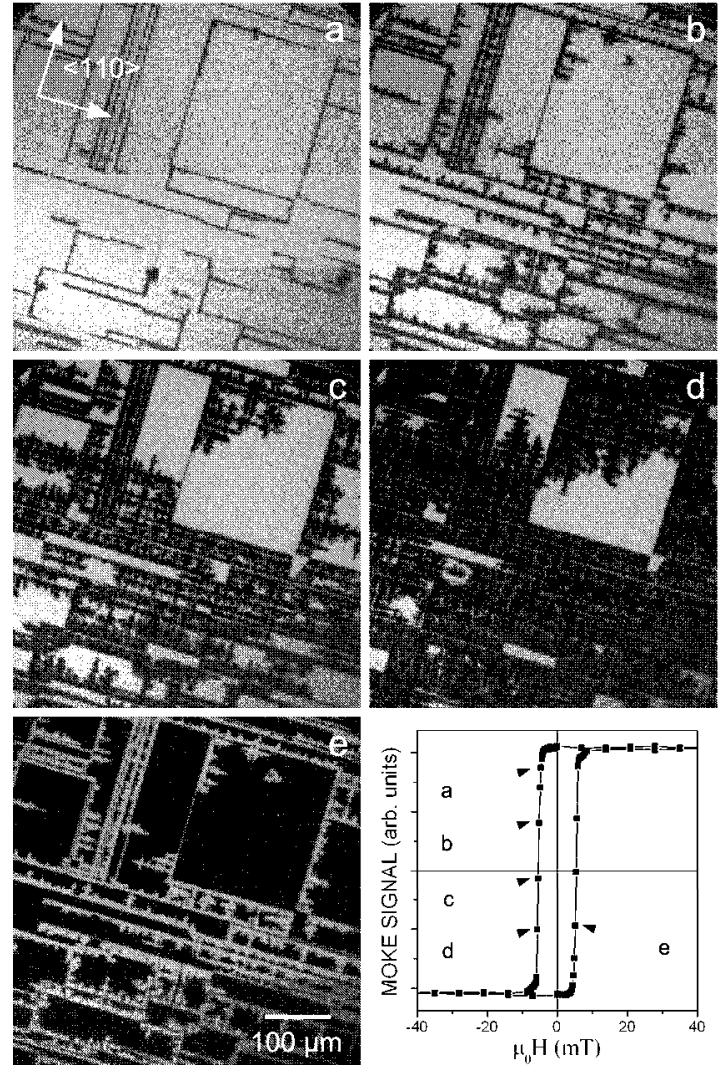

FIG. 5: Magneto-optical images showing the domain structure of the as-grown sample $93 \mathrm{~L} 06-a g$ at $30 \mathrm{~K}$. The corresponding field values are shown by arrows on the hysteresis cycle.

$<110>$ directions. Further side-branching and domain growth yield a grid-like pattern, as shown in Fig. 5 (c) and (d). It must be noted that some of the first reversed line domains grow over more than $100 \mu \mathrm{m}$ almost without side branching. In the course of magnetization reversal, they appear as strong barriers that prevent domain propagation in the perpendicular direction. These lines delimit large rectangular areas with high coercivity such as the one observed in the upper right part of all images in Fig. 5.

For sample 25M05-ag one clearly observes multiple nucleations (Fig. 6 (a)) followed by a dendritic-like growth along the $\langle 110\rangle$ directions (Fig. 6 (b)), which finally also results in a grid-like pattern (Fig. 6 (c) and (d)). In contrast with sample 93L06-ag, no long line domain along the $\langle 110\rangle$ directions is observed.

The comparison of images taken at the beginning of magnetization reversal for opposite values of the field (Fig. 5 (b) and (e), and Fig. 6 (b) and (e)) shows a high degree of reproducibility for nucleation sites and propagation path.

Figure 7 shows images of sample 25M05-ag obtained at a temperature where the easy-axis lies in the layer plane $(T=3 \mathrm{~K})$. The images are taken at field values close to one of the two hysteresis loops, as indicated by arrows

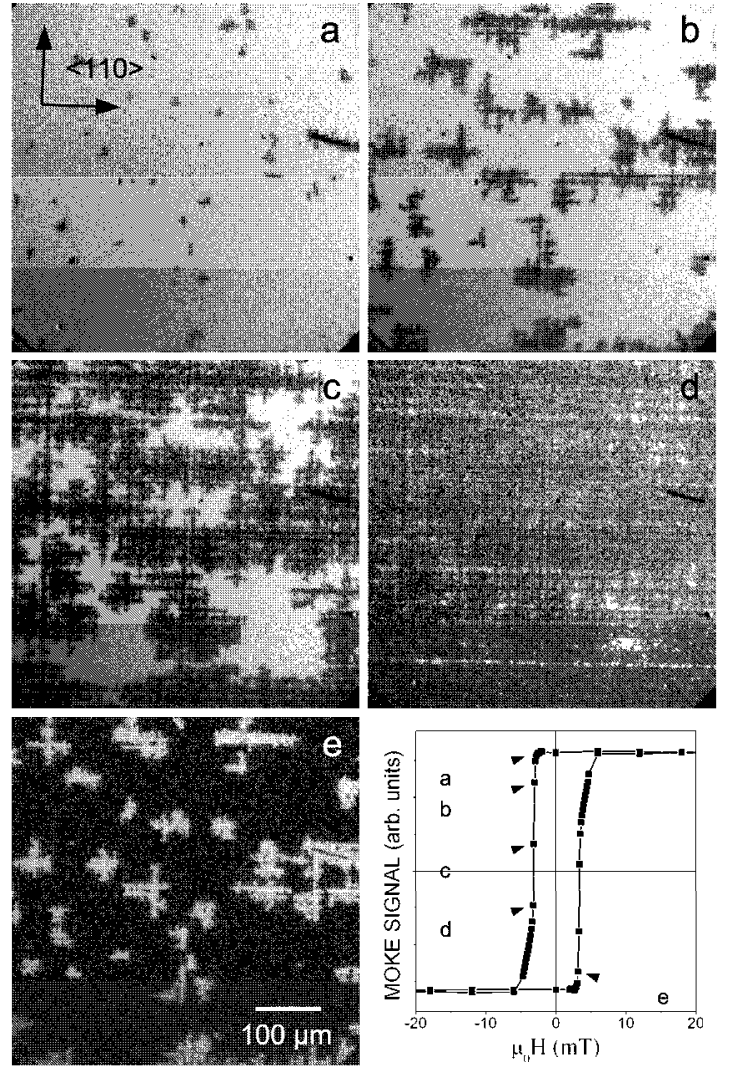

FIG. 6: Magneto-optical images showing the domain structure of the as-grown sample $25 \mathrm{M} 05-\mathrm{ag}$ at $30 \mathrm{~K}$. The corresponding field values are shown by arrows on the hysteresis cycle.

on the hysteresis cycle. When sweeping the applied field up to $\sim 25 \mathrm{mT}$, the magnetization acquires a small outof-plane component although no domain can be observed (Fig. 7 (a)). Above $\sim 25 \mathrm{mT}$, domains with out-of-plane magnetization develop and form a grid-like pattern along the $\langle 110\rangle$ directions (Fig. 7 (b) and (c)). When the magnetization switches back to the plane (Fig. 7 (d)) chains of dot-like domains with in-plane magnetization (white domains) appear in the black background of the out-of-plane magnetized layer. They form stripe-like domains. Noticeably, the domains that switch their magnetization back to the layer plane more easily (white domains in Fig. 7 (d) and (e)) appear to be the same as the last ones that switched their magnetization out of the plane when the field was swept up (white domains in Fig. $7(\mathrm{c}))$. This is consistent with the existence of spatial inhomogeneities of the uniaxial anisotropy energy that governs the switching of the magnetization from inplane to out-of-plane direction. From the images it is seen that these inhomogeneities occur on a length scale of a few $\mu \mathrm{m}$ or less.

It is worth noting that the domain pattern described here is strikingly different from the large-size domain pattern shown in Ref. $[22]$, for a sample with similar in-plane magnetization but grown on a GaAs buffer. We attribute 


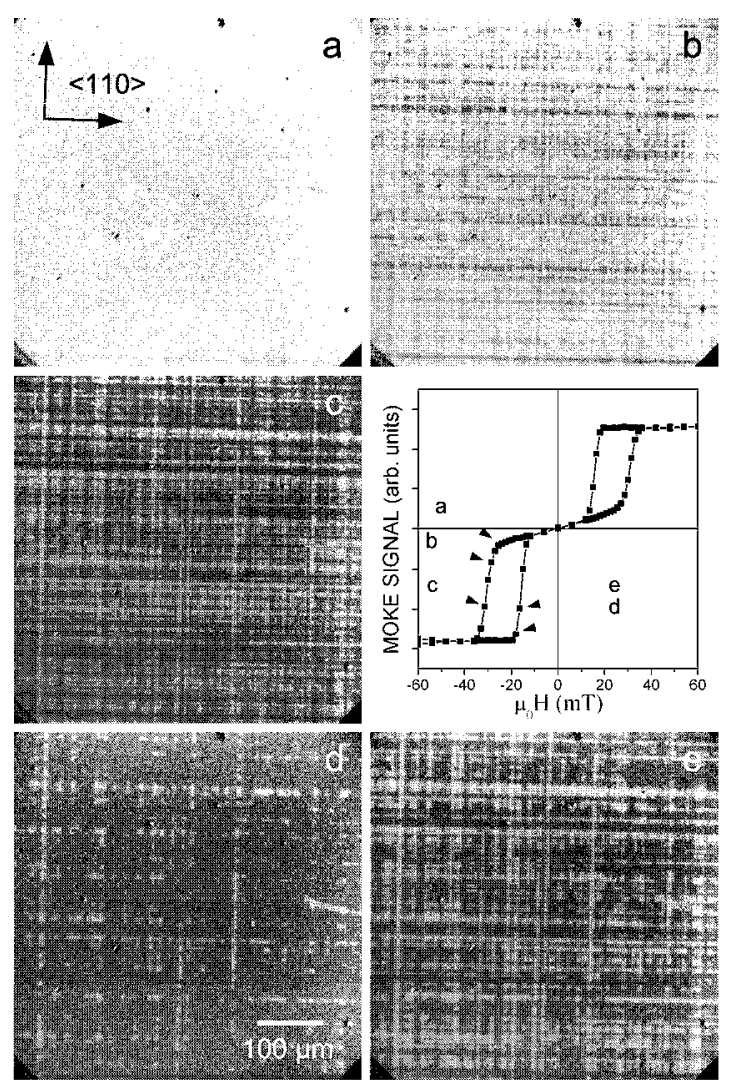

FIG. 7: Magneto-optical images showing the domain structure of the as-grown sample $25 \mathrm{M} 05$-ag at $3 \mathrm{~K}$. The easy magnetization axis lies in the sample plane. The corresponding field values are shown by arrows on the hysteresis cycle.

this difference to the influence of the (Ga,In)As buffer, which will be discussed in Sec. V.

\section{B. Annealed samples}

In this section we show the domain structure of annealed samples, and we compare it to that of as-grown samples. Magneto-optical images are shown in Fig. 8 and Fig. 9 for samples 93L06-a and 25M05-a, respectively. The images are taken at magnetization reversal, as indicated by the arrows on the cycles.

Let us consider the first steps of magnetization reversal for the as-grown and annealed samples. A comparison between Fig. 5(a) (sample 93L06-ag) and Fig. 8(a) (sample 93L06-a) on the one side, and Fig. 6(a) (sample 25M05-ag) and Fig. 9(a) (sample 25M05-a) on the other side, shows that annealing strongly decreases the density of nucleation sites. For sample 25M05-ag (Fig. 6(a)) one observes about 40 nucleation centers inside the field of view $(530 \mu \mathrm{m} \times 530 \mu \mathrm{m})$ but only one or two for the annealed sample (Fig. 9(a)). Let us note that the nucleation field does not change upon annealing, which makes this comparison meaningful. For sample 93L06-a, at the first stage of magnetization reversal, one observes fine stripes aligned along $<110>$ as for the as-grown sample.

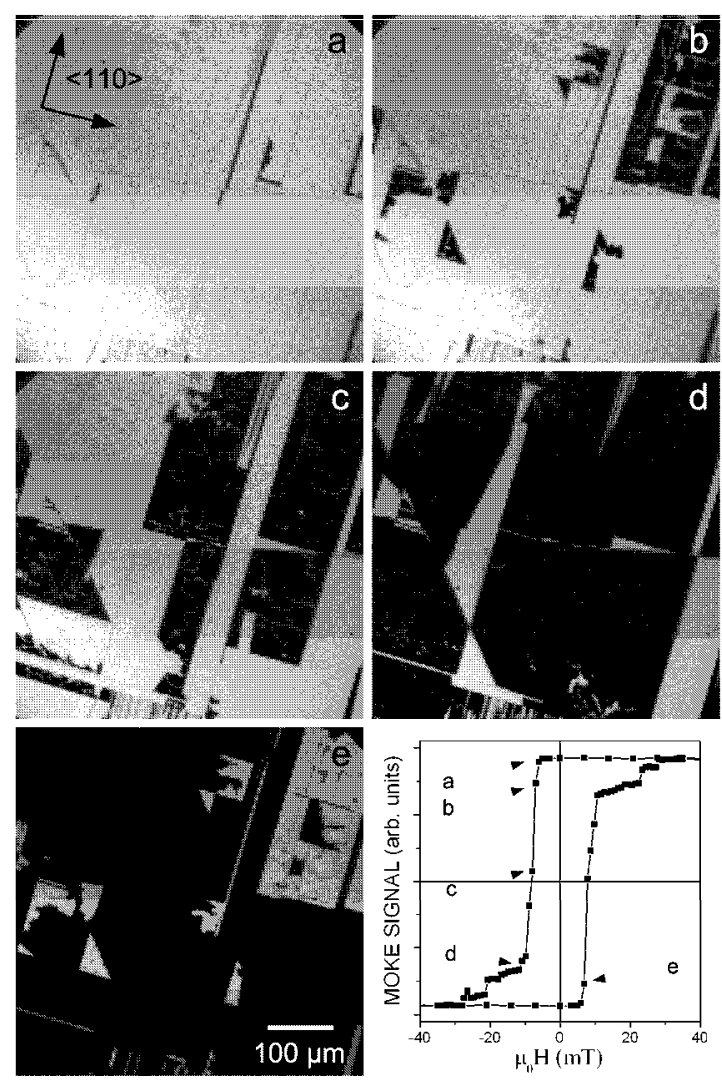

FIG. 8: Magneto-optical images showing the domain structure of the annealed sample $93 \mathrm{~L} 06$-a at $30 \mathrm{~K}$. The corresponding field values are shown by arrows on the hysteresis cycle.

As the field is further swept, nucleation is followed by domain wall motion. Annealing radically modifies the wall motion. This is more clearly observed for sample 25M05-a (Fig. 9). Domain walls are no more aligned along the $\langle 110\rangle$ axes. There is no preferential direction for their motion (Fig. 9(a) and (e)). However, domain wall motion is still not fully isotropic since it is locally hindered by pinning defects. Two kinds of defects are observed: point-like and linear defects. Linear defects, several hundreds of micrometers long, very efficiently delay or even stop domain wall propagation. This is sometimes observed as small steps in the hysteresis cycle between the coercive field and the saturation field. In sample 93L06-a two kinds of lines are observed. Some of them are aligned along the $\langle 110\rangle$ directions. They are very likely the same as those observed in the as-grown sample. They give rise to the block structure of the magnetic domains. Others, which are absent in the as-grown sample, have no specific orientation with respect to the crystallographic axes. In sample 25M05-a only this second kind of linear defects is observed. Most of these defects are straight but some are slightly curved. They are typically spaced by a few hundred micrometers.

Point-like defects appear in both samples, but are more clearly observed in sample 25M05-a (Fig. 9) ow- 


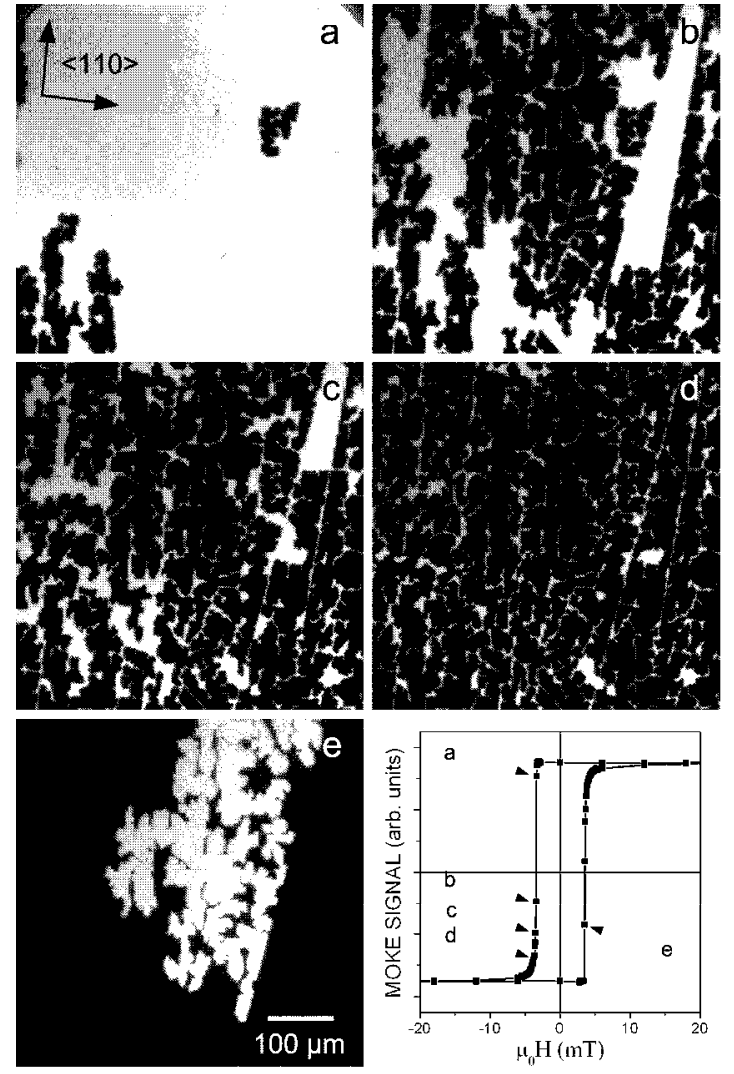

FIG. 9: Magneto-optical images showing the domain structure of the annealed sample $25 \mathrm{M} 05$-a at $30 \mathrm{~K}$. The corresponding field values are shown by arrows on the hysteresis cycle.

ing to better image contrast. They pin and fold up the walls at their passage, creating non-reversed narrow filaments. ${ }^{14,32}$ The filament width depends on temperature and applied field. In sample 93L06-a, inside the blocks delimited by the $\langle 110\rangle$ line defects, similar domain wall motion, with the presence of point-like pinning defects and filament domains, is observed (top right corner of Fig. 8(b) and (e)). For both samples, close to the ond of magnetization reversal, only some filaments and small areas nearby defects remain non-reversed. With further increase of the field they disappear one after the other.

The origin of the pinning defects is not well understood. Concerning the linear defects appearing after annealing, they do not originate from additional surface steps or undulations, as checked by AFM and interferential microscopy. Point-like pinning centers are located at the ends of the filaments, thereby making possible an estimation of their density $\geq 2-310^{5} \mathrm{~cm}^{-2}$. They could be associated with emerging dislocations ${ }^{14}$ or nanoclusters of $\mathrm{Mn}$ or MnAs. ${ }^{33}$

As for the as-grown samples, the position of the nucleation sites for annealed samples is very reproducible and the paths followed by the walls show many similarities from one field cycle to the next. This means that de- fects govern domain nucleation and domain wall motion. As already noted in Sec. III, annealing has little effect on the width of the hysteresis cycle. Despite the drastic reduction of nucleation centers for sample $25 \mathrm{M} 05$, the coercive field hardly changes. It remains two times smaller than for sample 93L06-a at $30 \mathrm{~K}$. This means that the coercive field mostly depends on defects that pin domain walls. We attribute the larger coercive field in samples 93L06-ag and 93L06-a to the higher density of linear defects aligned along the $\langle 110\rangle$ directions. Again, this has to be related to the larger indium content of the buffer layer and therefore to a larger density of dislocations.

In order to determine whether domain expansion depends of the value of the saturation magnetization $M_{s}$, we studied the temperature dependence of the domain structure. Since $M_{s(T \rightarrow 0)}$ is larger for annealed than for as-grown samples and $M_{s}$ decreases with the temperature, there are two temperatures $T_{1}$ and $T_{2}$ for which $M_{s}\left(T_{1}\right)_{\text {annealed }}=M_{s}\left(T_{2}\right)_{\text {as-grown }}$ with $T_{1}>T_{2}$. If domain expansion were $M_{s}$-dependent, with increasing temperature annealed samples should exhibit a change from the quasi-isotropic domain wall expansion towards the anisotropic and dendritic-like propagation characteristic of as-grown samples. Figure 10 shows domain structures for sample 25M05-a near the coercive field at $T=10$, 40,100 , and $120 \mathrm{~K}$. Domain expansion at all these temperatures is quite similar. This means that domain wall propagation does not depend in a direct manner on the value of the saturation magnetization $M_{s}(T)$, and hence on Mn concentration.

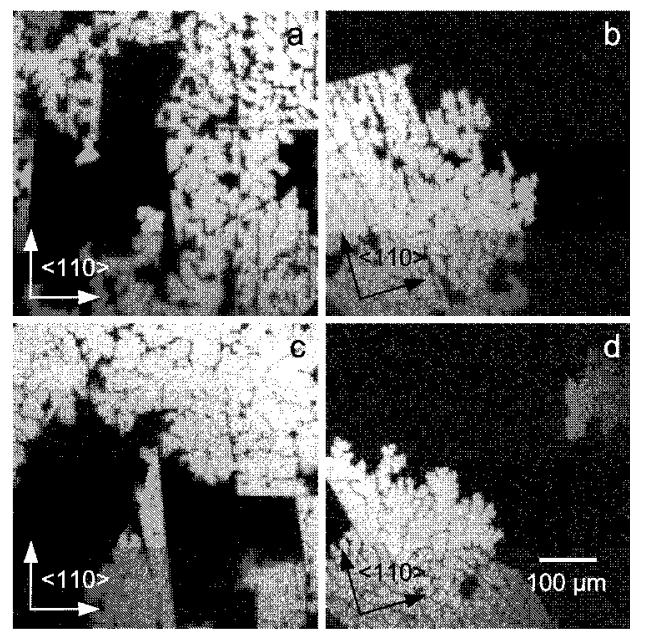

FIG. 10: Magneto-optical images of the magnetic domain structure at $10 \mathrm{~K}(\mathrm{a}), 40 \mathrm{~K}(\mathrm{~b}), 100 \mathrm{~K}(\mathrm{c})$ and $120 \mathrm{~K}(\mathrm{~d})$ for sample $25 \mathrm{M} 05-\mathrm{a}$, near the coercive field.

It must be noted that pinning is strong enough to prevent the system from reaching the demagnetized state expected at thermodynamical equilibrium. This state, with zero global magnetization at zero applied field, should exhibit the well-known stripe or bubble self-organized pattern. ${ }^{36}$ Using AC demagnetization, we searched for these patterns in sample $25 \mathrm{M} 05-\mathrm{a}$, which has the lowest 
density of linear-like pinning defects. Even with a field sweeping rate as low as $0.1 \mathrm{mT} \mathrm{s}^{-1}$, domain reorganization into bubble or stripe patterns was not observed. Therefore one should be very careful when using domain theory to extract material parameters. ${ }^{34}$

\section{DISCUSSION}

\section{A. High coercivity and polarized reflectivity}

a)

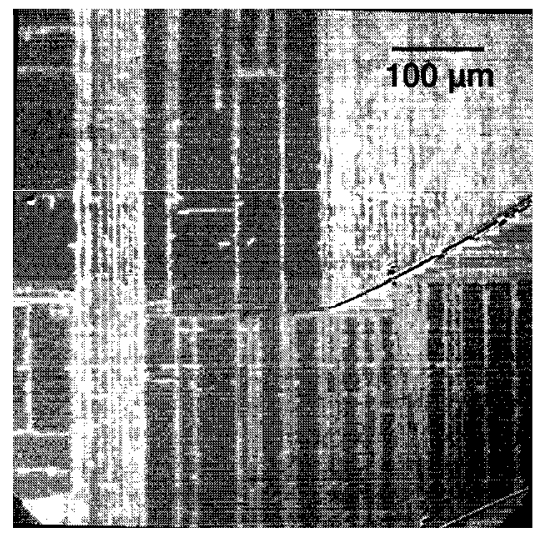

b)

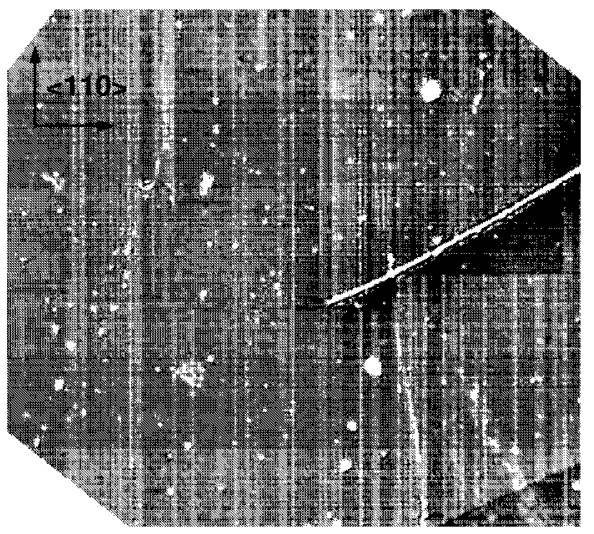

FIG. 11: MOKE magnetic image (a) and optical microscopy image (b) obtained at the same position on sample 93L06ag. The scale is the same for both images. For image (a) the applied field is $\mu_{0} H=9.8 \mathrm{mT}$ and the temperature 1.8 $\mathrm{K}$. Image (b) is obtained at room temperature with nearly crossed polarizer and analyzer, and one of the $\langle 100\rangle$ axes aligned with the polarizer. The contrast has been enhanced with respect to the raw image.

A clear correlation between the magnetic pattern (Fig. 11(a)) and the pattern revealed by polarized optical microscopy at room temperature (Fig. 11(b)) was observed for sample 93L06-ag. For this sample, the surface undulations of the cross-hatch pattern have an amplitude large enough to make the cross-hatch pattern observable by optical microscopy, of course with a spatial resolution limited by the optics. With nearly crossed polarizer and analyzer, and one of the $\langle 100\rangle$ directions of the sample aligned with the polarizer, some line domains ap- pear brighter or darker than the background (Fig. 11(b)). These lines are aligned along the $\langle 110\rangle$ directions and are irregularly spaced. The dark lines, which are perpendicular to the bright ones, appear mostly as closelyspaced lines in areas delimited by a defect or by straight boundaries (triangular areas in Fig. 11(b)). The contrast between the dark and bright lines is inverted when the sample is rotated by $90^{\circ}$ whereas the overall intensity of the image does not vary. These results are consistent with a small degree of linear polarization along $<110>$ of the light reflected or scattered by these lines.

The surface topography of the same area of the sample was observed by AFM. The bright lines seem to correspond to undulations with large ridge-groove amplitude. However not all the undulations with large amplitude appear as bright lines in the optical image.

The magnetic image (Fig. 11(a)) was taken near the coercive field. The field was cycled only between $+26.7 \mathrm{mT}$ and $-26.7 \mathrm{mT}$ within the major cycle. Although the magnetization appears to be saturated at these field values, some line domains with non-reversed magnetization still persist. These line domains have a larger coercivity than average. When the field is swept back and reversed, these domains grow first because they are already present: there is no nucleation barrier. Therefore, the magnetic domains appearing in this image correspond to areas with coercivity larger than average. We can conclude that there is a very clear correlation between these areas with larger coercivity and the lines with $<110\rangle$ polarization observed by optical microscopy. Similar optical images were observed for the annealed sample 93L06-a. However, for samples 25M05-ag and 25M05-a, the cross-hatch pattern was hardly observable using optical microscopy. Such dark and bright lines were not observed. Given the corrclation between the high-cocreivity line domains and the surface profile revealed by polarized optical microscopy for sample 93L06, we suspect that the larger In content of the (Ga,In)As buffer, leading to a high density of dislocations, and/or the thicker (Ga,Mn) As layer $(16 \%, 200 \mathrm{~nm}$, respectively) are detrimental for the magnetic homogeneity of the layer.

\section{B. Anisotropic domain growth}

In this section, we discuss the differences between asgrown and annealed samples regarding domain expansion at magnetization reversal. We consider the temperature range where all samples have perpendicular easy-axis. For the as-grown samples, the domain structure is characterized by strong in-plane anisotropy: domains grow along the $\langle 110\rangle$ directions. Anisotropic growth might originate from intrinsic effects such as anisotropic domain wall energy, or from extrinsic effects related to domain wall pinning or to spatial fluctuations of the magnetic properties of the layer.

Let us first discuss domain wall energy. The in-plane anisotropy was investigated by FMR for sample 25M05- 
ag at $30 \mathrm{~K}$. The resonance field is lower along the $<100>$ directions $(347 \mathrm{mT})$ than along the $<110\rangle$ ones $(399.8$ and $416.4 \mathrm{mT}$ ). This means that, among the hard axes in the sample plane, the $\langle 100\rangle$ directions are the softer ones. The same result was reported in Ref. [35] for a $(\mathrm{Ga}, \mathrm{Mn}) \mathrm{As} /(\mathrm{Ga}, \mathrm{In}) \mathrm{As}$ sample. Inside a domain wall (either a Bloch wall, a Néel wall or a twisted wall) the magnetic moments rotate by $180^{\circ}$, passing through an intermediate direction in the sample plane. In order to minimize the wall energy, the in-plane component of the magnetization at the wall center should be aligned along a $<100>$ axis. Therefore one expects the domain walls to be aligned along the $\langle 100\rangle$ directions. This is in contradiction with experimental observations that show domain expansion along the $\langle 110\rangle$ directions. It means that domain wall motion is very likely not governed by the small intrinsic in-plane magnetic anisotropy.

There is obviously a striking similarity between the grid-like pattern of magnetic domains observed by Kerr microscopy and the cross-hatch structure observed by AFM, although a quantitative comparison is made difficult by the different spatial resolutions of the two techniques. This raises the question of the interplay between surface topography and magnetic domain structure. The experimental observations suggest a segregation of pinning centers and/or a spatial modulation of the layer physical properties along the ridges and grooves of the cross-hatch pattern. Given the anisotropic domain expansion for as-grown samples, one could speculate that domain wall pinning centers are localized along the ridges and grooves. However, in annealed samples, domain expansion does not show any evidence for such localization. Firstly, a cartography of the point-like pinning centers (not shown here) was obtained for sample 25M05-a. It does not reveal any segregation along the $\langle 110\rangle$ directions. Secondly, since there is no clear preferential direction for domain wall motion, other pinning centers, which do not pin domain walls so strongly but might control domain wall motion in the creep regime, also do not segregate along the $\langle 110\rangle$ directions. These results suggest that, upon annealing, pinning centers would become inactive or be randomized. Given the low annealing temperature and the negligible effect of annealing on the width of the hysteresis cycle at low temperature, this process appears unlikely.

An alternative and appealing interpretation can be proposed, based on Ref. [37,38]. The authors developed a simulation of domain reversal in the presence of a spatial distribution of the uniaxial anisotropy energy. Interestingly the reversal behavior changes from nucleationdominant to wall-motion-dominant as the amplitude of anisotropy fluctuations decreases. Fluctuations of a few percent are enough to produce drastic changes of the magnetic pattern. ${ }^{37}$ Moreover, in the nucleationdominant regime, dendrite-like domains are formed. This model is in qualitative agreement with the characteristic features of magnetization reversal described in Sec. IV, i.e. nucleation-dominant and dendritic-like expansion for as-grown samples, wall-motion dominant for annealed samples. The existence of anisotropy energy fluctuations is consistent with the large linewidth of FMR spectra, typically 5 to 10 times larger for (Ga,Mn)As grown on $(\mathrm{Ga}, \mathrm{In}) \mathrm{As}$ than for $(\mathrm{Ga}, \mathrm{Mn}) \mathrm{As}$ grown on GaAs. In addition, the anisotropic domain wall expansion for as-grown samples suggests a spatial correlation between the uniaxial anisotropy energy fluctuations and the cross-hatch pattern. A qualitative explanation of the change of the magnetization reversal process upon annealing can be proposed: with the increase of the carrier concentration, the uniaxial anisotropy energy increases and the relative amplitude of its fluctuations decreases. The increase of the anisotropy energy is clearly established, at least for sample 25M05 where the magnetic easy-axis switches for in-plane to out-of plane below $20 \mathrm{~K}$ after annealing. Uniaxial anisotropy energy fluctuations can arise from fluctuations of strain, carrier concentration, or Mn concentration. The decrease of their relative amplitude can be expected from theoretical results ${ }^{1}$ but calculations with our sample parameters are needed to obtain quantitative estimations.

\section{CONCLUSION}

We have investigated the magnetic domain structure of $(\mathrm{Ga}, \mathrm{Mn})$ As epilayers grown with tensile strain on a ( $\mathrm{Ga}$,In) As buffer by MOKE microscopy. The general features are weakly temperature-dependent, provided that the magnetic easy-axis lies perpendicular to the plane. The domain structure and expansion are strikingly different from that reported in the literature for $(\mathrm{Ga}, \mathrm{Mn}) \mathrm{As}$ epilayers grown on a GaAs buffer. The cross-hatch pattern, arising from the (Ga.In)As buffer, appears to play an important role. It should be noted that similar investigations on $\mathrm{a}(\mathrm{Ga}, \mathrm{Mn}) \mathrm{As}$ layer grown on an indium rich buffer were published while this paper was in the reviewing process. ${ }^{39}$

Furthermore, as-grown and annealed samples exhibit a markedly different magnetization reversal process: (i) the density of nucleation sites decreases by more than one order of magnitude upon annealing; (ii) the expansion of reversed domains proceeds in a very different manner for as-grown and annealed samples, as summarized hereafter.

As-grown samples exhibit a strongly anisotropic domain growth. Domains expand as stripes along the $<110>$ directions with dendritic-like branching in the direction perpendicular to the stripes. This anisotropic expansion cannot be explained by in-plane anisotropy. We attribute it to the existence of spatial fluctuations of the uniaxial anisotropy energy along the ridges and grooves of the surface cross-hatch pattern.

For annealed samples, domain wall motion is much more isotropic, which is attributed to a decrease of uniaxial anisotropy fluctuations associated with the increase of the carrier density. However two kinds of defects are 
found to hinder free motion: point-like and linear defects. Point-like defects locally pin domain walls that fold up around them, leaving non-reversed filaments up to well beyond the coercive field. Their density is at least 2$310^{5} \mathrm{~cm}^{-2}$. Linear or slightly curved defects, hundreds of micrometers long, which appear upon annealing, exhibit various orientations with respect to the crystallographic axes. They very efficiently impede domain wall motion.

As a conclusion, magneto-optical imaging of magnetic domain patterns in (Ga,Mn)As epilayers grown on (Ga,In)As brings a new insight on the effect of annealing in perpendicular-axis (Ga,Mn)As. Annealing does not only increase the Curie temperature and the carrier density but also drastically modifies the magnetization reversal process. Fluctuations of the uniaxial anisotropy en- ergy, correlated with the surface cross-hatch pattern, may play a key role. Further theoretical investigations, including simulations of magnetic domain reversal in the presence of correlated fluctuations of the uniaxial anisotropy energy, would be of great interest to back up this interpretation.

\section{Acknowledgments}

We gratefully acknowledge the assistance of Emmanuelle Lacaze from Institut des Nanosciences de Paris, and Stéphane Flusil from Unité Mixte de Physique CNRS/Thalès, for AFM measurements.
1 T. Dietl, H. Ohno, and F. Matsukura, Phys. Rev. 63, 195205 (2001).

2 J. König, T. Jungwirth, and A. H. MacDonald, Phys. Rev. B 64, 184423 (2001).

3 T. Jungwirth, J. Sinova, J. Mašek, J. Kučera, A. H. MacDonald, Rev. Mod. Phys. 78, 809 (2006).

${ }^{4}$ P. Mahadevan, A. Zunger, and D. D. Sarma, Phys. Rev. Lett. 93, 177201 (2004).

${ }^{5}$ K. M. Yu, W. Walukiewicz, T. Wojtowicz, I. Kuryliszyn, X. Liu, Y. Sasaki, and J. K. Furdyna, Phys. Rev. B 65, 201303 (2002).

${ }^{6}$ T. Hayashi, Y. Hashimoto, S. Katsumoto, and Y. Iye, Appl. Phys. Lett. 78, 1691 (2001).

7 S. J. Potashnik, K. C. Ku, S. H. Chun, J. J. Berry, N. Samarth, and P. Schiffer, Appl. Phys. Lett. 79, 1495 (2001).

${ }^{8}$ M. B. Stone, K. C. Ku, S. J. Potashnik, B. L. Sheu, N. Samarth, and P. Schiffer, Appl. Phys. Lett. 83, 4568 (2003).

9 B. S. Sørensen, P. E. Lindelof, J. Sadowski, R. Mathieu and P. Svedlindh, Appl. Phys. Lett. 82, 2287 (2003).

${ }^{10}$ K. W. Edmonds, K. Y. Wang, R. P. Campion, A. C. Neumann, C. T. Foxon, B. L. Gallagher, and P. C. Main, Appl. Phys. Lett. 81, 3010 (2002).

11 K. W. Edmonds, K. Y. Wang, R. P. Campion, A. C. Neumann, N. R. S. Farley, B. L. Gallagher, and C. T. Foxon, Appl. Phys. Lett. 81, 4991, (2002).

12 K.W. Edmonds, P. Bogusławski, K.Y.Wang, R. P. Campion, S. N. Novikov, N. R. S. Farley, B. L. Gallagher, C.T. Foxon, M. Sawicki, T. Dietl, M. Buongiorno Nardelli, and J. Bernholc, Phys. Rev. Lett. 92, 037201 (2004).

13 A. Pross, S. Bending, K. Edmonds, R. P. Campion, C. T. Foxon, and B. Gallagher, J. Appl. Phys. 95, 3225 (2004).

${ }^{14}$ L. Thevenard, L. Largeau, O. Mauguin, G. Patriache, A Lemaître, N. Vernier, and J. Ferré, Phys. Rev. B 73, 195331 (2006).

15 Y. Ohno, D. K. Young, B. Beschoten, F. Matsukura, H. Ohno, D. D. Awschalom, Nature 402, 790 (1999).

${ }^{16}$ D. Chiba, Y. Sato, T. Kita, F. Matsukura, and H. Ohno, Phys. Rev. Lett. 93, 216602 (2004).

17 M. Elsen, O. Boulle, J.-M. George, H. Jaffrès, R. Mattana, V. Cros, A. Fert, A. Lemaitre, R. Giraud, and G. Faini,
Phys. Rev. B 73, 035303 (2006).

18 M. Yamanouchi, D. Chiba, F. Matsukura, and H. Ohno, Nature 428, 539 (2004).

19 K. Y. Wang, R. P. Campion, K. W. Edmonds, M. Sawicki, T. Dietl, C. T. Foxon, and B. L. Gallagher, 27th Intern. Conf. on the Physics of Semiconductors, AIP Proceedings 772, 333 (2005).

${ }^{20}$ M. Sawicki, F. Matsukura, A. Idziaszek, T. Dietl, G. M. Schott, C. Ruester, C. Gould, G. Karczewski, G. Schmidt, and L. W. Molenkamp, Phys. Rev. B 70, 245325 (2004).

21 L. Thevenard, L. Largeau, O. Mauguin, and A. Lemaître, Appl. Phys. Lett. 87, 182506 (2005).

${ }^{22}$ U. Welp, V. K. Vlasko-Vlasov, X. Liu, J. K. Furdyna, T.Wojtowicz, Phys. Rev. Lett. 90, 157206 (2003).

23 T. Shono, T. Hasegawa, T. Fukumura, F. Matsukura and H. Ohno, Appl. Phys. Lett 77, 1363 (2000).

${ }^{24}$ T. Fukumura, T. Shono, K. Inaba, T. Hasegawa, H. Koinuma, F. Matsukura, H. Ohno, Physica E 10, 135 (2001).

${ }^{25}$ F. Glas, G. Patriarche, L. Largeau, and A. Lemaitre, Phys. Rev. Lett. 93, 086107 (2004)

26 A. M. Andrews, J. S. Specka, A. E. Romanov, M. Bobeth, and W. Pompe, J. Appl. Phys. 91, 1933 (2002).

27 H. Chen, Y. K. Li, C. S. Peng, H. F. Liu, Y. L. Liu, Q. Huang, J. M. Zhou, and Qi-Kun Xue, Phys. Rev. B 65, 233303 (2002).

28 O. Yastrubchak, T. Wosiński, J. Z. Domagala, E. Lusakowska, T. Figielski, B. Pécz and A. L. Tóth, J. Phys.: Condens. Matter 16, S1S8 (2004).

29 C. Gourdon, V. Jeudy, M. Menant, D. Roditchev, Le Anh Tu, E. L. Ivchenko, and G. Karczewski, Appl. Phys. Lett. 82, $230(2003)$.

${ }^{30}$ X. Liu, W.L. Lim, L.V. Titova, T. Wojtowicz, M. Kutrowski, K.J. Yee, M. Dobrowolska, J.K. Furdyna, S.J. Potashnik, M.B. Stone, P. Schiffer, I. Vurgaftman, J.R. Meyer, Physica E 20, 370 (2004).

31 D. Hrabovsky, E. Vanelle, A. R. Fert, D. S. Yee, J. P. Redoules, J. Sadowski, J. Kanski, and L. Ilver, Appl. Phys. Lett. 81, 2806 (2002).

32 A. Dourlat, C. Gourdon, V. Jeudy, C. Testelin, K. Khazen, J.L. Cantin, H.J. von Bardeleben, L. Thevenard, and A. Lemaitre, to be published in IEEE Trans. Magn. 
${ }^{33}$ K. Y. Wang, M. Sawicki, K. W. Edmonds, R. P. Campion, A. W. Rushforth, A. A. Freeman, C. T. Foxon, and B. L. Gallagher, T. Dietl, Appl. Phys. Lett 88022510 (2006).

${ }^{34}$ T. Dietl, J. König, A. H. MacDonald, Phys. Rev. B 64, 241201 (2001).

${ }^{35}$ X. Liu, Y. Sasaki, and J. K. Furdyna, Phys. Rev. B 67 , 205204 (2003).

36 A. Hubert and R. Schäfer, Magnetic domains, Springer, Berlin, 2000.
37 Sug-bong Choe and Sung-chul Shin, IEEE Trans. Mag. $36,3167(2000)$.

38 E. A. Jagla, Phys. Rev B 72, 094406 (2005).

${ }^{39}$ K. Y. Wang, A. W. Rushforth, V. A. Grant, R.P. Campion, K. W. Edmonds, C. R. Staddon, C. T. Foxon, B. L. Gallagher, J. Wunderlich, and D. A. Williams, J. Appl. Phys. 101, 106101 (2007). 